\title{
Hepatitis B virus-Associated Intrahepatic Cholangiocarcinoma Has Distinct Clinical, Pathological and Radiological Characteristics:
} A Systematic Review

\author{
Harry Ho Man Ng${ }^{1}$, Nathanelle Khoo ${ }^{2}$, Jyothirmayi Velaga ${ }^{3}$, Joe Yeong ${ }^{4,5}$, Jin Yeo $\mathrm{Teo}^{2}$, \\ Brian K.P Goh ${ }^{1,2}$, Albert Su-Chong Low ${ }^{3}$, Chung Yip Chan ${ }^{1,2}$, Tony Kiat Hon Lim", Ser Yee Lee ${ }^{1,2}$
}

'Duke-National University of Singapore (NUS), Medical School, Singapore

${ }^{2}$ Department of Hepatopancreatobiliary and Transplant Surgery, Singapore General Hospital, Singapore

${ }^{3}$ Department of Diagnostic Radiology, Singapore General Hospital, Singapore

${ }^{4}$ Department of Anatomical Pathology, Division of Pathology, Singapore General Hospital, Singapore

${ }^{5}$ Institute of Molecular Cell Biology (IMCB), Agency of Science, Technology and Research $(A *$ STAR), Singapore

\section{ABSTRACT}

Intrahepatic cholangiocarcinoma (ICC) is a rare hepatic malignancy with a poor prognosis. It is known that Hepatitis B is both a risk factor and a prognosis factor for ICC. However, little is known about the clinical, pathological and radiological characteristics of ICC patients with hepatitis B virus (HBV) infection. A systematic review of relevant literature was performed using PubMed and EMBASE databases. HBV-associated ICC revealed several unique and interesting features, it was found to have higher alpha-fetoprotein (AFP), cancer antigen 19-9 (CA 19-9) levels, capsule formation and cirrhosis, but associated with lower lymph node metastasis. Radiologically, it was shown that some of the HBV-associated ICC tend to mimic atypical radiological features of HCC. Pathologically, it was found that $H B x$ protein gene expression was associated with HBV-associated ICC, and that hepatic progenitor cells may be an alternative origin for HBV-associated ICC. In conclusion, HBV-associated ICC has distinct clinical, pathological and radiological characteristics, but further research is needed to further understand the pathogenesis of the disease.

Key words: hepatitis B virus, intrahepatic cholangiocarcinoma, clinical characteristics, radiological features, pathogenesis

\section{INTRODUCTION}

Intrahepatic cholangiocarcinoma (ICC) is a rare but aggressive malignancy originating from the bile ducts inside the liver, representing $10-15 \%$ of all primary hepatic malignancies (1). As ICC is initially asymptomatic, there are challenges in obtaining an early diagnosis, resulting in a low resection rate and a poor prognosis, with a median survival of less than 24 months, and 1- and 2-year
Corresponding author:

Ser Yee Lee, MBBS, MMED,MSc, FRCS, FAMS,

Department of Hepatopancreatobiliary and Transplant Surgery, Singapore General Hospital, Singapore Academia, 20 College Road, Singapore 169856

Tel: +65-66325564

E-mail: lee.ser.yee@singhealth.com.sg

\section{Abbreviations:}

ICC - Intrahepatic cholangiocarcinoma; HBV - hepatitis B virus;

AFP - alpha-fetoprotein;

CA 19-9 - cancer antigen 19-9;

HR - hazard ratio;

$\mathrm{Cl}$ - confidence interval;

OS - overall survival;

DFS - disease-free survival;

ALT - aminotransferase;

AST - aspartate aminotransferase;

GGT - gamma-glutamyl transferase;

AFP - alpha-fetoprotein;

CK19 - cytokeratin 19;

CEUS - contrast-enhanced ultrasound; LI-RADS - Liver Imaging Reporting and Data System;

MAPK - mitogen-activated proteinbase; HPCs - Hepatic progenitor cells; ADC - apparent diffusion coefficient; DWI - diffusion weighted imaging; NCAM - neural cell adhesion molecule; BCL-2 - B-cell lymphoma 2; 
survival rates of $25 \%$ and $13 \%$, respectively $(2,3)$.

The liver flukes Clonorchis sinensis and Opisthorchis viverrini, as well as chronic biliary inflammation conditions such as primary sclerosing cholangitis, Caroli's disease, hepatolithiasis are established major risk factors of the disease $(4,5)$. Other risk factors include biliary duct cysts, toxins such as thorotrast (thorium dioxide)(6).

Hepatitis B is a potentially life-threatening liver infection most prevalent in Asia Pacific and sub-Saharan African regions in the world (7). Hepatitis B virus (HBV) has been recently shown to be a significant risk factor for development of ICC. In a meta-analysis of studies evaluating the risk of HBV infection and ICC in 2012, the pooled risk ratio $(2.66 ; 95 \% \mathrm{Cl}=1.97$ to 2.60$)$ was significantly higher for those individuals who were HBVpositive compared with those who were HBV-negative (8). In a recent large scale population based study in the United States, Hepatitis B was stated as a risk factor of ICC with an odds ratio of $2.97(95 \% \mathrm{Cl}=1.97$ to 4.46$)$ (9).

It has been suggested that HBV-associated ICC may have unique features such as distinct clinical, pathological and radiological characteristics and HBV-associated ICC may have a different pathobiology when compared to the non-HBV-associated ICC. The literature is limited in this area, therefore, we performed a systematic review to better evaluate the clinical, pathological and the radiological characteristics of HBV-associated ICC.

\section{METHODS}

The study was performed in line with the recommendations of the Preferred Reporting Items for Systematic Reviews and Meta-Analyses (PRISMA) statement (10).

\section{Literature Search}

We performed a systematic search of the pertinent literature, the databases of EMBASE and Medline were reviewed from Jan 1998 to Sept 2018 for relevant English language studies. The literature search used the following terms: Hepatitis B, cholangiocarcinoma and their synonyms and abbreviations (Table 1). We also examined the reference lists of published review articles and meta-analyses to identify other eligible studies.

\section{Study Selection}

One investigator (HHN) performed the initial searching, extraction of data, and quality assessment, which was subsequently reviewed by all other involved authors. Studies were assessed on the basis of their title or abstract and those studies which appeared to meet the eligibility criteria were selected for full text review. We sought studies reporting at least one of the following aspects of HBV-associated ICC: prognosis and clinical, radiological, and pathological characteristics. Small series assessing fewer than 20 patients, as well as studies without clinical, radiological or pathological data were excluded. Studies reporting on hetereogenous series of patients with intrahepatic and other types of cholangiocarcinoma (e.g., hilar cholangiocarcinoma, mixed cholangio-hepatocellular carcinoma) were also excluded. In addition, studies not differentiating between Hepatitis B or C virus were excluded. Previous systematic review and meta-analysis were reviewed, but not included in the final analysis. Conference abstracts that did not proceed to publication in peerreviewed journals were not included in the review.

\section{Data extraction}

Relevant in formation from eligible publications were extracted. All publications included were reviewed by the senior author (SYL). For each study, the following data were captured: (1) authorship and year of publication; (2) the study population and size; (3) the study design; (4) the clinical parameters, including age, gender, clinical stage; (5) clinical characteristics (if applicable) including prognosis [hazard ratio (HR) and 95\% confidence interval $(\mathrm{Cl})$ of HBV infection for overall survival (OS) and disease-free survival (DFS)]; (7) pathological characteristics (if applicable) including biomarkers; and (8) radiological characteristics (if applicable).

\section{RESULTS}

\section{Literature Search and study characteristics}

The publication search and selection process is illustrated in Fig. 1. 732 potentially eligible papers were retrieved in the initial search. After review of the titles and abstracts, 646 publications were excluded. 86 full articles were captured, among which 53 were finally

Table 1 - Terms used in literature search

\begin{tabular}{ll}
\hline Hepatitis B & Cholangiocarcinoma \\
\hline HBV & bile duct neoplasms \\
\hline $\mathrm{CHB}$ & $\mathrm{ICC}$ \\
\hline & Biliary tract cancers \\
\hline
\end{tabular}




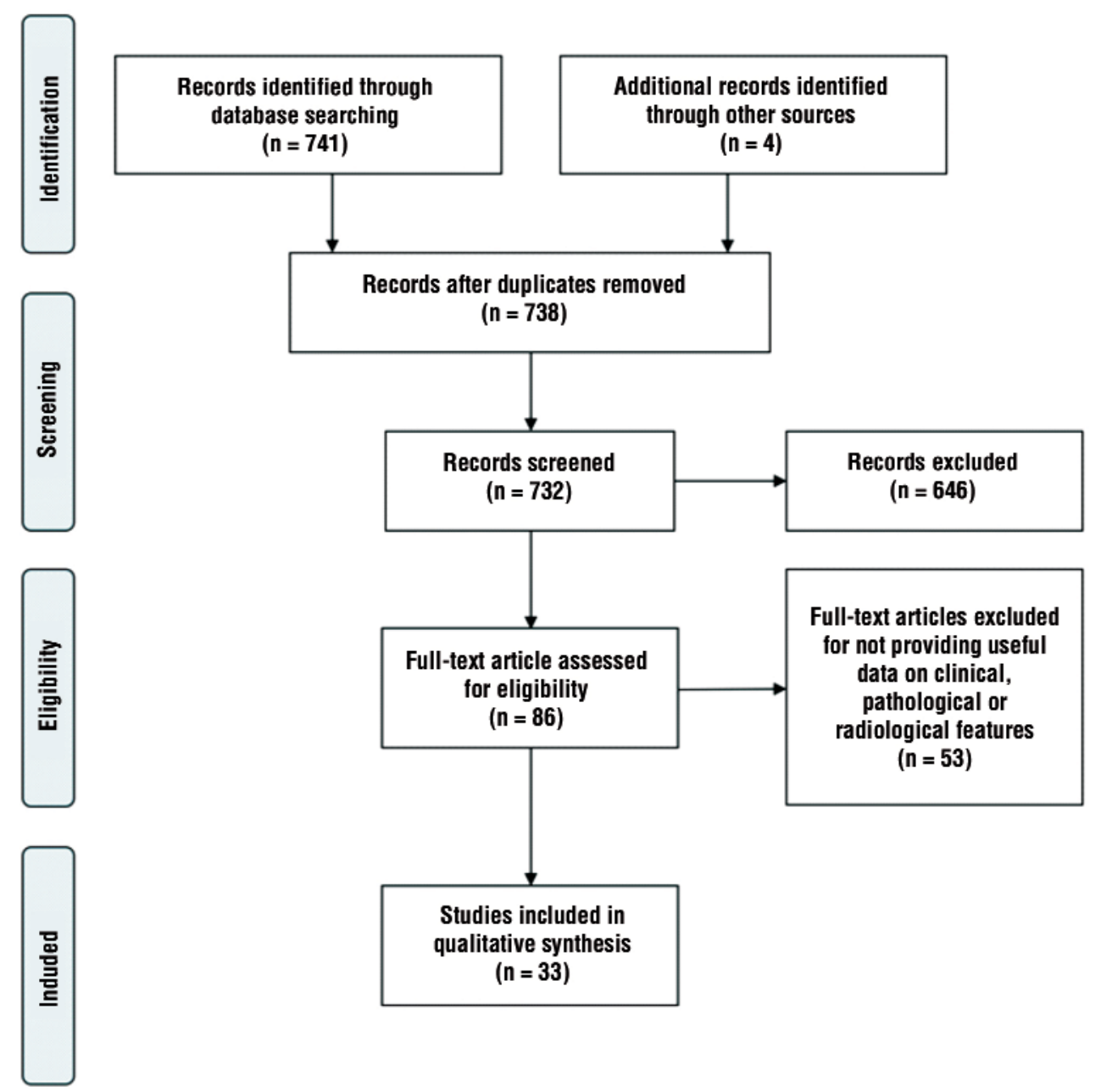

Figure 1 - PRISMA flow diagram on literature review strategy

excluded due to the insufficient information on HBV status, or lacking relevant data on clinical, pathological or radiological features. Finally, our review enrolled a total of 33 studies. Of these publications chosen for further assessment, 20 investigated the correlation of HBV-associated and non-HBV-associated ICC and their clinical characteristics, and 7 studied the relationship of HBV and its pathological features, and 10 correlated $\mathrm{HBV}$ virus status and the radiological features.

The main characteristics of each enrolled study are summarized in Table 2. The publications included in our study were published between 1991 and 2017. In total, 20 studies have reported clinical characteristics between HBV and non-HBV associated ICC. For clinical characteristics, 10 studies enrolled less than 100 cases, and 10 studies enrolled more than 100 cases. The mean age of the enrolled patients ranged from 51 to 62 years in these studies.

\section{Clinical characteristics of $H B V$-associated ICC}

Clinical characteristics of HBV-associated ICC has been established from multiple studies. Firstly, for biochemical markers, there was a study reporting HBV infection was associated with higher alanine aminotransferase (ALT) levels, and 2 studies with higher aspartate aminotransferase (AST) levels $(11,12)$. One study has reported the HBV-associated group having lower gamma-glutamyl transferase (GGT) levels, but other studies showed no differences in GGT levels between HBV-associated and non-HBV-associated ICCs (11-14). The rest of the studies did not find any significant differences liver enzymes levels between the 2 groups (13-16). All studies that reported bilirubin levels has similar levels between HBVassociated and non-HBV-associated ICCs (11,12,14-16). All studies that reported alpha-fetoprotein (AFP) levels concluded that HBV-associated ICC is associated with 
Table 2 - Main characteristics of all the studies included in the systematic review

\begin{tabular}{|c|c|c|c|c|c|c|c|}
\hline Study & Study country & Patient number & Mean age & Gender (M/F) & Clinical staging & Chacteristics & Study design \\
\hline Zhou et al. (11) & China & 317 & $53.1 \pm 10.5$ & $223 / 94$ & NA & Clinical & Retrospective \\
\hline Zhou et al. (12) & China & 155 & $\begin{array}{l}55.0 \pm 10.7 \\
(27.0-76.0)\end{array}$ & $102 / 53$ & NA & $\begin{array}{c}\text { Clinical, } \\
\text { Pathological }\end{array}$ & Retrospective \\
\hline Ahn et al. (15) & Korea & 292 & $59 \pm 10$ & $196 / 96$ & I-IV & Clinical & Retrospective \\
\hline Tao et al. (13) & China & 97 & $\begin{array}{c}58.6 \\
(37-78)\end{array}$ & $59 / 38$ & I-IV & Clinical & Retrospective \\
\hline Wang et al. (16) & China & 606 & $53.7(44.5-62.3)$ & $401 / 205$ & I-IV & Clinical & Retrospective \\
\hline Wu et al. (14) & China & 138 & 55 & $107 / 31$ & I-IV & Clinical & Retrospective \\
\hline Peng et al. (17) & China & 62 & $\mathrm{NA}$ & $\mathrm{NA}$ & NA & Clinical & Retrospective \\
\hline Jeong et al. (18) & China & 106 & $60(35-82)$ & $62 / 44$ & I-IV & Clinical & Retrospective \\
\hline Jiang et al. (19) & China & 76 & $51.0(40.0-60.0)$ & $53 / 23$ & I-IV & Clinical & Retrospective \\
\hline Liu et al. (20) & China & 81 & $59.0(30.0-76.0)$ & $48 / 33$ & NA & Clinical & Retrospective \\
\hline Zhang et al. (21) & China & 40 & $56.0(34.0-74.0)$ & $24 / 16$ & I-IV & $\begin{array}{c}\text { Clinical, } \\
\text { Pathological }\end{array}$ & Retrospective \\
\hline Luo et al. (22) & China & 1333 & $54.1 \pm 10.9$ & $912 / 421$ & I-IV & Clinical & Retrospective \\
\hline Pan et al. (23) & China & 72 & $53.26 \pm 11.05$ & $29 / 43$ & I-III & Clinical & Retrospective \\
\hline Asayama et al. (24) & Japan & 67 & $62.0(33.0-83.0)$ & $36 / 31$ & NA & $\begin{array}{c}\text { Clinical, } \\
\text { Pathological }\end{array}$ & Retrospective \\
\hline Zhang et al. (25) & $\begin{array}{l}\text { USA, Europe, } \\
\text { Australia, Asia }\end{array}$ & 883 & $59(45-69)$ & $497 / 386$ & I-IV & Clinical & Retrospective \\
\hline Zhang et al. (26) & China & 127 & $55.5 \pm 11.8$ & $102 / 25$ & IIIV & Clinical & Retrospective \\
\hline Uenishi et al. (27) & Japan & 35 & $61.0(35.0-83.0)$ & 11,24 & IIIIV & Clinical & Retrospective \\
\hline Horino et al. (28) & Japan & 31 & $65(26-81)$ & $21 / 13$ & NA & Clinical & Retrospective \\
\hline Li et al. (29) & China & 283 & $55.0(18.0-79.0)$ & $174 / 109$ & I-IV & Clinical & Prospective \\
\hline Wang et al. (30) & China & 110 & NA & NA & NA & Pathological & Retrospective \\
\hline Zhou et al. (31) & China & 54 & $49(23-73)$ & $36 / 18$ & NA & Pathological & Retrospective \\
\hline Huang et al. (33) & China & 71 (out of 612 ) & $55(29-77)$ & $51 / 20$ & NA & Radiological & Retrospective \\
\hline Kang et al. (34) & China & 50 & $62.3(42-76)$ & $41 / 9$ & NA & Radiological & Retrospective \\
\hline Kim et al. (35) & Korea & 26 (out of 105) & $60.6(40-75)$ & $22 / 4$ & NA & Radiological & Retrospective \\
\hline Li et al. (36) & China & 84 & $50(24-75)$ & $66 / 18$ & NA & Radiological & Retrospective \\
\hline Sheng et al. (37) & China & 28 (out of 162) & 60 & $19 / 9$ & NA & Radiological & Retrospective \\
\hline Galassi et al. (40) & Italy & 34 & $64.2 \pm 8.4$ & $16 / 8$ & NA & Radiological & Retrospective \\
\hline Li et al.(41) & China & 64 & $52.84 \pm 11.58$ & NA & NA & Radiological & Retrospective \\
\hline Yuan et al. (42) & China & 96 & NA & NA & NA & Radiological & Retrospective \\
\hline lavarone et al. (38) & Italy & 32 & $62(45-81)$ & $22 / 10$ & NA & Radiological & Retrospective \\
\hline Rimola et al. (39) & Spain & 25 & $62(49-79)$ & $17 / 8$ & NA & Radiological & Retrospective \\
\hline
\end{tabular}

higher AFP levels $(11-14,16,17)$. All but one study that reported cancer antigen 19-9 (CA 19-9) levels reported that HBV-associated ICC is associated with relatively lower CA 19-9 levels compared to non-HBV-associated ICCs (11-17).

For tumour characteristics, among the 6 studies that reported cirrhosis status, 4 studies showed that there was a higher prevalence of liver cirrhosis amongst HBV-associated ICC patients (11-14,16,17). Three studies also stated that there were higher levels of capsule formation but Peng et al. reported no statistical significance between the 2 groups $(11,12,14,17)$. For lymph node metastasis, 2 studies reported lower lymph node metastasis for HBV-associated ICC, but 6 other studies reported no statistical significance between the 2 groups (11-18). All studies reported no difference in vascular invasion (11-18) or perineural invasion $(11,12$, 15) between the 2 groups. There was conflicting evidence for tumour differentiation. Zhou et al. reported that HBV-associated ICC was associated with poor differentiation, and Wu et al. reported nonHBV-associated ICC was associated with better differentiation, whereas all other studies reported no statistical significance between the 2 groups (11-18). Peng et al. also reported that HBV-associated ICC was associated with higher neutrophilic infiltration, but this finding was not reported in any other studies (17).

In addition, several studies evaluated the correlation between the presence of HBV infection and the prognosis of patients with ICC (Table 3). Some of these studies suggested HBV infection as a favourable prognostic factor in patients with ICC $(12,14,16,19-24)$. 
Table 3 - Studies showing survival outcomes in HBV-associated ICC

\begin{tabular}{|c|c|c|c|}
\hline Study & $\begin{array}{l}\text { No. of patients }(\mathrm{n}): \\
\text { HBV-ICC/non-HBV-ICC }\end{array}$ & OS HR $(95 \% \mathrm{Cl})^{*}$ & $\begin{array}{l}\text { Prognosis of HBV-ICC } \\
\text { compared to non-HBV-ICC }\end{array}$ \\
\hline Zhou et al.(12) & $29 / 11$ & $0.46(0.19,1.09)^{\wedge}$ & Favourable \\
\hline Ahn et al.(15) & $37 / 255$ & $0.51(0.22,1.21)$ & No difference \\
\hline Wang et al. (16) & $519 / 87$ & $0.37(0.26,0.53)$ & Favourable \\
\hline Wu et al. (14) & $46 / 81$ & $0.49(0.34,0.71)$ & Favourable \\
\hline Jiang et al. (19) & $34 / 42$ & $0.88(0.72,1.04)^{\wedge}$ & Favourable \\
\hline Liu et al. (20) & $99 / 41$ & $0.49(0.34,0.71)$ & Favourable \\
\hline Zhang et al. (21) & $29 / 11$ & $0.46(0.19,1.09)^{\wedge}$ & Favourable \\
\hline Luo et al. (22) & $608 / 752$ & $0.73(0.63,0.85)$ & Favourable \\
\hline Pan et al. (23) & $34 / 38$ & $0.426(0.232-0.785)$ & Favourable \\
\hline Asayama et al. (24) & $8 / 55$ & $0.64(0.70,0.83)$ & Favourable \\
\hline Zhang et al. (25) & $201 / 682$ & Not significant when adjusted & No difference \\
\hline Zhang et al. (26) & $46 / 81$ & $0.86(0.58,1.27)$ & No difference \\
\hline Uenishi et al. (27) & $3 / 29$ & $0.70(0.22,2.24)$ & No difference \\
\hline Horino et al. (28) & $8 / 23$ & Not significant & No difference \\
\hline Li et al. (29) & $174 / 109$ & $0.88(0.59,1.290)$ & No difference \\
\hline
\end{tabular}

*if applicable; ^ significant in univariate analysis

However, there were a few studies that reported HBV infection has no significant impact on the survival outcome of patients with ICC after hepatic resection, especially after propensity score matching $(15,25-29)$.

\section{Pathological characteristics of $\mathrm{HBV}$-associated ICC}

There has been postulation that $H B x$ gene is key for the pathogenesis of ICC for HVB-associated cholangiocarcinoma. Wang et al. discovered the presence of $\mathrm{HBX}$ gene in different liver cancers, including cholangiocarcinoma (30). This has sparkled the interest of how the gene is involved in the pathogenesis of the disease. Zhou et al. (31) reported that $H B x$ expression was found in most of the cholangiocarcinoma tissues, and it was associated with elevated serum AFP. However, they also reported that only $33.3 \%$ of HBV-related ICCs exhibited p53 protein expression and it was not correlated with $\mathrm{HBx}$ gene expression (31).

Another study has shown that there was an increased co-expression of neural cell adhesion molecule (NCAM) and B-cell lymphoma 2 (BCL-2) in HBV-associated ICC (24). NCAM is a potential hepatic progenitor cell marker (32), which brings the speculation on a possible alternative origin of ICC. Two other studies also showed a lower cytokeratin 19 (CK19) expression on HBV-associated ICC $(11,12)$.

Other prognosis markers have also been evaluated in some studies. In one study, HBV-associated ICC was associated with lower human epidermal growth factor receptor 2 (HER2) expression, where HEP1 and MUC1 expression was not associated with HBV positivity (16). These data suggested the potential role of such markers on carcinogenesis and possibly prognosis.

\section{Radiological characteristics of $\mathrm{HBV}$-associated ICC}

The radiological characteristics of cholangiocarcinoma are well established. Mass forming cholangiocarcinoma are typically hypoattenuating relative to the normal liver tissues in pre-contrast scans, and heterogeneous peripheral early enhancement with gradual enhancement centrally in the postcontrast scans $(33,34)$. This is in contrast to other primary liver cancers such as HCC, which typically show arterial phase hyperenhancement and washout in the portal venous and delayed phases.

However, the radiological characteristics of ICCS in patients with hepatitis B and cirrhosis are not clear, as there are no studies that directly compare the radiological characteristics of HBV-associated ICC and non-HBV-associated ICC (Table 4). However, multiple studies observed arterial enhancement with washout in the delayed phase in a small portion of ICC tumours (6-15.3\%) on multiphase contrast Computed Tomography (CT) and Magnetic Resonance (MR) imaging, similar to the washout pattern of HCC (33-37) (Fig. 2). Most of the studies has associated this finding with liver cirrhosis in ICC, but HBV infection is present in most of the patients in these studies as well. They concluded that the arterial enhancement pattern of ICCs seems to be affected by the underlying liver disease, i.e. 
Table 4 - Summary of the characteristics of HBV-associated ICC compared to non-HBV-associated ICC

\begin{tabular}{|c|c|c|c|}
\hline & HBV-ICC & Non-HBV-ICC & Reference \\
\hline \multicolumn{4}{|l|}{ Biochemical markers: } \\
\hline ALT & $\uparrow$ & $\downarrow$ & $\begin{array}{c}(11) \\
(12-16)\end{array}$ \\
\hline AST & $\uparrow$ & 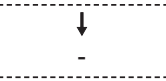 & $\begin{array}{l}(11,12) \\
(13-16)\end{array}$ \\
\hline GGT & $\downarrow$ & $\uparrow$ & $\begin{array}{c}(14) \\
(11-13)\end{array}$ \\
\hline Bilirubin & - & - & $(11,12,14-16)$ \\
\hline AFP & $\uparrow$ & $\downarrow$ & $(11-14,16,17)$ \\
\hline CA $19-9$ & $\downarrow$ & $\uparrow$ & $\begin{array}{c}(11-14,16,17) \\
(15)\end{array}$ \\
\hline \multicolumn{4}{|l|}{ Tumour characteristics: } \\
\hline Cirrhosis & $\uparrow$ & $\downarrow$ & $\begin{array}{c}(11,12,14,17) \\
(13,16)\end{array}$ \\
\hline Capsule formation & $\uparrow$ & $\downarrow$ & $\begin{array}{c}(11,12,14) \\
(17)\end{array}$ \\
\hline Lymph Node Metastasis & $\downarrow$ & $\uparrow$ & $\begin{array}{c}(12,14) \\
(11,13,15-18)\end{array}$ \\
\hline Vascular Invasion & $\begin{array}{ll}-- \\
\cdots\end{array}$ & - & $(11-18)$ \\
\hline Perineural Invasion & $\begin{array}{ll}-- \\
\cdots\end{array}$ & - & $(11,12,15)$ \\
\hline Tumour differentiation & $\begin{array}{l}\downarrow \\
\uparrow \\
-\end{array}$ & $\downarrow$ & $\begin{array}{c}(12) \\
(14) \\
(11,13,15-18)\end{array}$ \\
\hline Neutrophilic infiltration & $\uparrow$ & $\downarrow$ & (17) \\
\hline Prognosis & $\uparrow$ & $\downarrow$ & $\begin{array}{c}(12,14,16,19-24) \\
(15,25-29)\end{array}$ \\
\hline \multicolumn{4}{|l|}{ Pathological characteristics: } \\
\hline NCAM and bcl-2 co-expression & $\uparrow$ & $\downarrow$ & $(24)$ \\
\hline CK19 expression & $\downarrow$ & $\uparrow$ & $(11,12)$ \\
\hline HER2 expression & $\downarrow$ & $\uparrow$ & $(16)$ \\
\hline HEP1 expression & - & - & $(16)$ \\
\hline MUC1 expression & - & - & (16) \\
\hline
\end{tabular}

Legend: $\uparrow=$ increased/ better, $\downarrow$ = decreased/ worse, - = not significant difference and/or limited evidence, ALT = Alanine Aminotransferase, AST = Aspartate Aminotransferase, GGT = Gamma-Glutamyl Transferase, AFP = Alpha-Fetoprotein,

CA 19-9 = Cancer Antigen 19-9, NCAM = Neural cell adhesion molecule, bcl-2 = B-cell lymphoma 2, HER2 = human epidermal growth factor receptor 2

cirrhosis and/or HBV positivity. Interestingly, two studies that had no ICC tumours displaying "HCC-like" washout pattern did not include any HBV-positive cases in their patient cohort $(38,39)$. In fact, the aetiology of those patients having cirrhosis-related ICC were mostly due to Hepatitis $C$ infection or alcohol consumption $(38,39)$. It is possible to postulate that HBV-associated ICC displays radiological features less typical of non-HBV associated ICC, but this warrants further investigation.

For contrast-enhanced ultrasound (CEUS), there were a few studies that reported a significant portion of patients with HBV-associated ICC having heterogeneous hyperenhancement with early washout, which was then thought to mimic HCC (40-42). However, the recent CEUS LI-RADS (Liver Imaging Reporting and Data System) published by the American College of Radiologists has recognised early washout on CEUS as being more typical for ICC than HCC which more typically show later and milder washout (43). The different washout behaviour between contrast agents for CT/MRI and that for CEUS is that the former accumulate in the interstitial spaces of tumours such as ICC in the late phase, whilst CEUS contrast stays only in the liver/tumour sinusoids resulting in marked and early washout (43). These earlier studies did not use the strict newer criteria of differentiation between HCC and ICC on CEUS, so their conclusions may not be accurate.

\section{DISCUSSION}

This is the first systematic review that explored the differences between clinical, pathological and radiological characteristics of ICC with and without HBV 


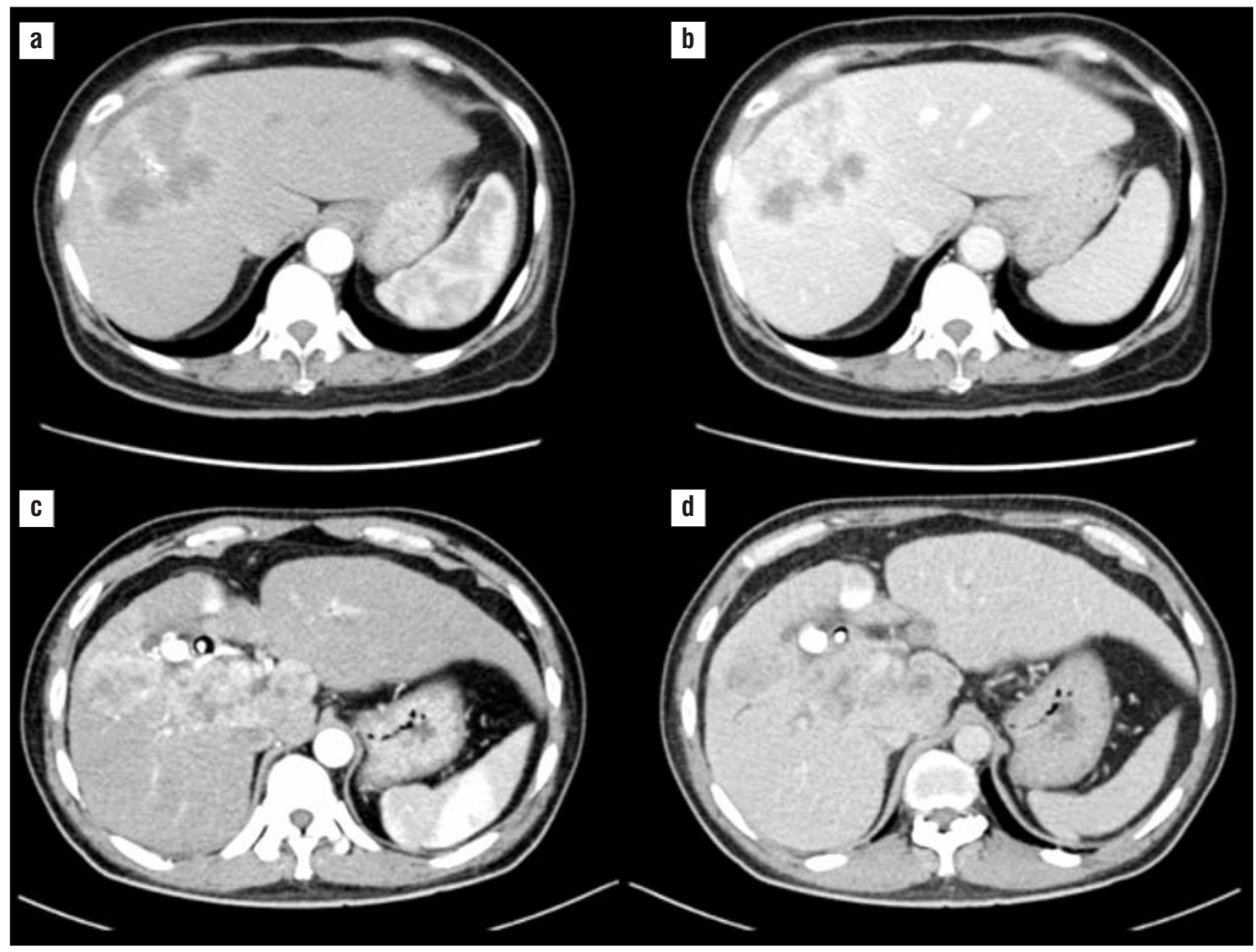

Figure 2 - (Top) Contrasted CT scan of a non-HBV-associated ICC patient showing (a) hypoenhancing tumour with peripheral arterial enhancement on the arterial phase, and (b) progressive centripetal enhancement on the venous phase. (Bottom) In contrast,contrasted CT scan of a HBV-associated ICC patient showing (c) early arterial enhancement followed by (d) rapid washout on the portal venous phase

infection, whereas previous studies hadonly focused largely on prognosis and other characteristics. From the systematic review, it suggests that there are some clinical characteristics of HBV-associated ICC that may be distinct from non-HBV-associated ICC. Specifically, HBV-associated ICCS may have a higher AFP and CA 19-9 levels, tend to have tumour capsule formation, cirrhosis and a lower rate of lymph node metastasis. Two large meta-analyses have shown that HBV was associated with better prognosis, which is consistent with the majority of the studies included in this systematic review, but this association warrants further prospective investigation $(44,45)$.

For pathological characteristics, it was demonstrated that $H B x$ gene was expressed in cholangiocarcinoma tissues in HBV-positive patients (30). Additional research using in vivo zebrafish showed that the pSmad3L oncogenic pathway was activated in $H B X-$ induced ICC (46). Moreover, phosphorylation of p38 mitogen-activated proteinbase (MAPK) and p44/42 mitogen-activated protein kinase (ERK1/2) was observed in the pathogenesis of ICC in the zebrafish model, indicating the association with transforming growth factor beta 1 (TGF- $\beta 1$ ) signalling pathway in HBV-associated ICC. This is similar to HCC, where HBV would disrupt the TGF- $\beta 1$ signalling through different pathways and act as an oncogene (47-49).

Using a cell culture model, Zou et al. reported that $H B x$ transfection induces human telomerase reverse transcriptase (hTERT) mRNA expression in human cholangiocytes in cell culture, causing cell immortality. These non-human studies provided additional information and suggested that $H B x$ may directly be involved in the carcinogenesis of cholangiocytes, which acts as an alternative pathway (50).

Hepatic progenitor cells (HPCs) are bipotential cells residing in normal liver. They possess the potential to differentiate toward the hepatocytic or biliary phenotype. As reservoir cells, HPCs were shown to be activated in a wide range of liver diseases and in conditions such as submassive necrosis, chronic viral hepatitis, and fatty liver disease (51-55). Cancer progression is thus often accompanied by HPC activation, but mechanisms initiated by injured hepatocytes remain still elusive.

Traditionally, it has been thought that ICC was 
caused by intrahepatic biliary epithelial cells. However, recently it has been debated that ICC could originate from multiple cell types. Some studies have shown that cholangiocarcinoma can originate from HPCs in some animal models $(56,57)$. Moreover, in some recent transcriptomic profiling analyses for liver cancers, it has been reported that some ICC subtypes with poor prognosis would present with hepatic stem-like gene signatures (58).

However, the proportion of ICC cases with a stem cell origin remains unclear. For Hepatitis $B$, increased expression of NCAM in human HBV-associated ICC tumours suggested that hepatic progenitor cells play an important role in the pathogenesis of the cancer (24). In a study focusing on cholangiocellular carcinoma, a subset of intrahepatic cholangiocarcinoma, a high level of expression of NCAM was reported in HBV-associated ICC, and thus some have postulated HPCs as a possible origin of ICC (59).

Studies have also investigated on the genome sequencing of ICC. The tumour pathogenesis of ICC is often heterogeneous, with multiple overlapping and lower-penetrance driver genes and a diverse signalling pathway. In a recent genetic study on ICC, it was found that KRAS mutation was predominately enriched in HBV-negative ICCs compared to HBV-positive cases (60). The same study also suggested that KRAS mutation was associated with poorer prognosis, thus the association of HBV-associated ICC with better prognosis could be due to the difference in mutation signatures.

In addition, a separate study has postulated a p53-mediated pathway being a main contributor to the tumori genesis of ICC if the patient is viral hepatitis positive (61). HBV-associated hepatocellular carcinoma (HCC) has also a similar inactivation of p53-mediated cell cycle pathway for a substantial number of patients, which may arise on non-cirrhotic livers $(62,63)$. Some other studies have shown increased expression of $\mathrm{N}$-cadherin and cholangiocellular differentiation in viral hepatitis, but $\mathrm{N}$-cadherin expression did not appear to be correlated with prognosis in ICC.

Changes of the tumour immune microenvironment is important in the pathogenesis of cancer, especially in virus-mediated cancers such as HCC, lymphoma and ICC. Current studies indicate that the neutrophil to lymphocyte ratio (NLR) may be a useful biomarker of long-term outcomes in patients with cholangiocarcinoma (64-66), but not much is known about how HBV affects the tumour immune microenvironment in ICC. Studies have shown that the HBV-associated HCC immune microenvironment was more immunosuppressive and exhausted than the non-HBV-associated HCC microenvironment, but not much is known for the immunological microenvironment in $\mathrm{HBV}$-associated ICCS (67). The chronic inflammation and changes in immunological microenvironment induced by HBV infection may be important in the pathogenesis for HBV-associated ICC, and further research is needed in exploring such hypotheses.

Early radiological diagnosis is important for the management for ICC. Misdiagnosis is not ideal as treatment between HCC and ICC can be very different. Some patients with HBV-associated ICC have arterial enhancement with washout in the delayed phase in CT/MR imaging, which is not seen in non-HBVassociated ICCS (Fig. 2). This phenomenon would be attributable to the changes in circulatory patterns due to portal hypertension, but there is limited information available on these possible changes in the literature. One way to differentiate between HBV-positive ICCS and HCCs is the use of apparent diffusion coefficient (ADC) derived from a mono-exponential model using diffusion weighted imaging (DWI) in MRI, but this method requires specific DWI imaging and is subject to further validation (68). Further research is needed to understand more on distinct radiological patterns on ICC tumours under the influence of HBV infection and cirrhosis.

\section{CONCLUSION}

In conclusion, we have shown that HBV-associated ICC has potentially distinct clinical, pathological and radiological characteristics compared to non-HBVassociated ICC as their pathobiology may be diverse. Moreover, multiple studies showed a possible origin of hepatic progenitor cells specific to HBV-associated ICC, thus it is plausible that ICC and HCC may hold common disease process for carcinogenesis under the effects of HBV (59). Future biological studies, especially focusing on the tumour microenvironment and radiological patterns are warranted to better understand biological mechanisms and behaviours of HBV-associated ICC, so as to improve diagnosis and help the establishment of specific subtype-targeting treatments.

\section{Conflict of Interest}

The authors declare that the research was conducted in the absence of any commercial or financial relationships that could be construed as a potential conflict of interest. 


\section{Funding}

This research was supported by AM-ETHOS DukeNUS Medical Student Fellowship Award (AM-ETHOS01/ FY2018/10-A10).

\section{REFERENCES}

1. Gatto M, Bragazzi MC, Semeraro R, Napoli C, Gentile R, Torrice A, et al. Cholangiocarcinoma: update and future perspectives. Dig Liver Dis. 2010;42(4):253-60.

2. Farley DR, Weaver AL, Nagorney DM. "Natural history" of unresected cholangiocarcinoma: patient outcome after noncurative intervention. Mayo Clin Proc. 1995;70(5):425-9.

3. Patel T. Worldwide trends in mortality from biliary tract malignancies. BMC Cancer. 2002;2:10.

4. Kurathong S, Lerdverasirikul P, Wongpaitoon V, Pramoolsinsap C, Kanjanapitak A, Varavithya W, et al. Opisthorchis viverrini infection and cholangiocarcinoma. A prospective, case-controlled study. Gastroenterology. 1985;89(1):151-6.

5. Lim MK, Ju YH, Franceschi S, Oh JK, Kong HJ, Hwang SS, et al. Clonorchis sinensis infection and increasing risk of cholangiocarcinoma in the Republic of Korea. Am J Trop Med Hyg. 2006;75(1):93-6.

6. Zhu AX, Lauwers GY, Tanabe KK. Cholangiocarcinoma in association with Thorotrast exposure. J Hepatobiliary Pancreat Surg. 2004; 11(6):430-3.

7. MacLachlan JH, Cowie BC. Hepatitis B virus epidemiology. Cold Spring Harb Perspect Med. 2015;5(5):a021410.

8. Li M, Li J, Li P, Li H, Su T, Zhu R, et al. Hepatitis B virus infection increases the risk of cholangiocarcinoma: a meta-analysis and systematic review. J Gastroenterol Hepatol. 2012;27(10):1561-8.

9. Petrick JL, Yang B, Altekruse SF, Van Dyke AL, Koshiol J, Graubard $\mathrm{Bl}$, et al. Risk factors for intrahepatic and extrahepatic cholangiocarcinoma in the United States: A population-based study in SEERMedicare. PLoS One. 2017;12(10):e0186643.

10. Moher D, Liberati A, Tetzlaff J, Altman DG, Group P. Preferred reporting items for systematic reviews and meta-analyses: the PRISMA statement. BMJ. 2009;339:b2535.

11. Zhou H, Wang H, Zhou D, Wang H, Wang Q, Zou S, et al. Hepatitis $B$ virus-associated intrahepatic cholangiocarcinoma and hepatocellular carcinoma may hold common disease process for carcinogenesis. Eur J Cancer. 2010;46(6):1056-61.

12. Zhou HB, Wang H, Li YQ, Li SX, Wang H, Zhou DX, et al. Hepatitis B virus infection: a favorable prognostic factor for intrahepatic cholangiocarcinoma after resection. World J Gastroenterol. 2011; 17(10): 1292-303.

13. Tao LY, He XD, Xiu DR. Hepatitis B virus is associated with the clinical features and survival rate of patients with intrahepatic cholangiocarcinoma. Clin Res Hepatol Gastroenterol. 2016; 40(6):682-7.

14. Wu ZF, Yang N, Li DY, Zhang HB, Yang GS. Characteristics of intrahepatic cholangiocarcinoma in patients with hepatitis $B$ virus infection: clinicopathologic study of resected tumours. J Viral Hepat 2013;20(5):306-10.

15. Ahn CS, Hwang S, Lee YJ, Kim KH, Moon DB, Ha TY, et al. Prognostic impact of hepatitis $B$ virus infection in patients with intrahepatic cholangiocarcinoma. ANZ J Surg. 2018;88(3):212-7.

16. Wang $Q$, Li J, Lei Z, Wu D, Si A, Wang K, et al. Prognosis of Intrahepatic Cholangiocarcinomas with HBV Infection is Better than Those with Hepatolithiasis After R0 Liver Resection: A Propensity Score Matching Analysis. Ann Surg Oncol. 2017;24(6):1579-87.

17. Peng NF, Li LQ, Qin X, Guo Y, Peng T, Xiao KY, et al. Evaluation of risk factors and clinicopathologic features for intrahepatic cholangiocarcinoma in Southern China: a possible role of hepatitis B virus. Ann Surg Oncol. 2011;18(5):1258-66.

18. Jeong S, Cheng Q, Huang L, Wang J, Sha M, Tong Y, et al. Risk stratification system to predict recurrence of intrahepatic cholangiocarcinoma after hepatic resection. BMC Cancer. 2017;17(1):464.
19. Jiang BG, Ge RL, Sun LL, Zong M, Wei GT, Zhang YJ. Clinical parameters predicting survival duration after hepatectomy for intrahepatic cholangiocarcinoma. Can J Gastroenterol. 2011;25(11):603-8.

20. Liu RQ, Shen SJ, Hu XF, Liu J, Chen LJ, Li XY. Prognosis of the intrahepatic cholangiocarcinoma after resection: hepatitis $B$ virus infection and adjuvant chemotherapy are favorable prognosis factors. Cancer Cell Int. 2013;13(1):99.

21. Zhang L, Cai JQ, Zhao JJ, Bi XY, Tan XG, Yan T, et al. Impact of hepatitis $B$ virus infection on outcome following resection for intrahepatic cholangiocarcinoma. J Surg Oncol. 2010;101(3):233-8.

22. Luo X, Yuan L, Wang Y, Ge R, Sun Y, Wei G. Survival outcomes and prognostic factors of surgical therapy for all potentially resectable intrahepatic cholangiocarcinoma: a large single-center cohort study. J Gastrointest Surg. 2014;18(3):562-72.

23. Pan QX, Su ZJ, Zhang JH, Wang CR, Ke SY. Glasgow Prognostic Score predicts prognosis of intrahepatic cholangiocarcinoma. Mol Clin Oncol. 2017;6(4):566-74.

24. Asayama $\mathrm{Y}$, Aishima $\mathrm{S}$, Taguchi $\mathrm{K}$, Sugimachi $\mathrm{K}$, Matsuura $\mathrm{S}$, Masuda K, et al. Coexpression of neural cell adhesion molecules and bcl-2 in intrahepatic cholangiocarcinoma originated from viral hepatitis: relationship to atypical reactive bile ductule. Pathol Int. 2002:52(4):300-6.

25. Zhang XF, Chakedis J, Bagante F, Beal EW, Lv Y, Weiss M, et al. Implications of Intrahepatic Cholangiocarcinoma Etiology on Recurrence and Prognosis after Curative-Intent Resection: a MultiInstitutional Study. World J Surg. 2018;42(3):849-57.

26. Zhang GW, Lin JH, Qian JP, Zhou J. Identification of risk and prognostic factors for patients with clonorchiasis-associated intrahepatic cholangiocarcinoma. Ann Surg Oncol. 2014;21(11):3628-37.

27. Uenishi T, Nagano H, Marubashi S, Hayashi M, Hirokawa F, Kaibori $\mathrm{M}$, et al. The long-term outcomes after curative resection for massforming intrahepatic cholangiocarcinoma associated with hepatitis C viral infection: a multicenter analysis by Osaka Hepatic Surgery Study Group. J Surg Oncol. 2014;110(2):176-81.

28. Horino K, Beppu T, Komori H, Masuda T, Hayashi H, Okabe H, et al. Evaluation of mass-forming intrahepatic cholangiocarcinoma with viral hepatitis. Hepatogastroenterology. 2012;59(116):1217-9.

29. Li T, Qin LX, Zhou J, Sun HC, Qiu SJ, Ye QH, et al. Staging, prognostic factors and adjuvant therapy of intrahepatic cholangiocarcinoma after curative resection. Liver Int. 2014;34(6):953-60.

30. Wang WL, London WT, Feitelson MA. Hepatitis $B \times$ antigen in hepatitis B virus carrier patients with liver cancer. Cancer Res. 1991; 51(18):4971-7.

31. Zhou YM, Cao L, Li B, Zhang XZ, Yin ZF. Expression of HBx protein in hepatitis $B$ virus-infected intrahepatic cholangiocarcinoma. Hepatobiliary Pancreat Dis Int. 2012;11(5):532-5

32. Tsuchiya A, Kamimura H, Tamura Y, Takamura M, Yamagiwa S, Suda $\mathrm{T}$, et al. Hepatocellular carcinoma with progenitor cell features distinguishable by the hepatic stem/progenitor cell marker NCAM. Cancer Lett. 2011:309(1):95-103.

33. Huang B, Wu L, Lu XY, Xu F, Liu CF, Shen WF, et al. Small Intrahepatic Cholangiocarcinoma and Hepatocellular Carcinoma in Cirrhotic Livers May Share Similar Enhancement Patterns at Multiphase Dynamic MR Imaging. Radiology. 2016;281(1):150-7.

34. Kang Y, Lee JM, Kim SH, Han JK, Choi BI. Intrahepatic massforming cholangiocarcinoma: enhancement patterns on gadoxetic acid-enhanced MR images. Radiology. 2012;264(3):751-60.

35. Kim SJ, Lee JM, Han JK, Kim KH, Lee JY, Choi BI. Peripheral massforming cholangiocarcinoma in cirrhotic liver. AJR Am J Roentgenol. 2007;189(6):1428-34.

36. Li R, Cai P, Ma KS, Ding SY, Guo DY, Yan XC. Dynamic enhancement patterns of intrahepatic cholangiocarcinoma in cirrhosis on contrastenhanced computed tomography: risk of misdiagnosis as hepatocellular carcinoma. Sci Rep. 2016;6:26772.

37. Sheng RF, Zeng MS, Rao SX, Ji Y, Chen LL. MRI of small intrahepatic mass-forming cholangiocarcinoma and atypical small hepatocellular carcinoma $(</=3 \mathrm{~cm})$ with cirrhosis and chronic viral hepatitis: a comparative study. Clin Imaging. 2014;38(3):265-72.

38. lavarone M, Piscaglia F, Vavassori S, Galassi M, Sangiovanni A, Venerandi $L$, et al. Contrast enhanced CT-scan to diagnose intrahepatic cholangiocarcinoma in patients with cirrhosis. J Hepatol. 
2013;58(6):1188-93.

39. Rimola J, Forner A, Reig M, Vilana R, de Lope CR, Ayuso C, et al Cholangiocarcinoma in cirrhosis: absence of contrast washout in delayed phases by magnetic resonance imaging avoids misdiagnosis of hepatocellular carcinoma. Hepatology. 2009;50(3):791-8.

40. Galassi M, lavarone M, Rossi S, Bota S, Vavassori S, Rosa L, et al. Patterns of appearance and risk of misdiagnosis of intrahepatic cholangiocarcinoma in cirrhosis at contrast enhanced ultrasound. Liver Int. 2013;33(5):771-9.

41. Li R, Zhang X, Ma KS, Li XW, Xia F, Zhong H, et al. Dynamic enhancing vascular pattern of intrahepatic peripheral cholangiocarcinoma on contrast-enhanced ultrasound: the influence of chronic hepatitis and cirrhosis. Abdom Imaging. 2013;38(1): 112-9.

42. Yuan MX, Li R, Zhang XH, Tang CL, Guo YL, Guo DY, et al. Factors Affecting the Enhancement Patterns of Intrahepatic Cholangiocarcinoma (ICC) on Contrast-Enhanced Ultrasound (CEUS) and their Pathological Correlations in Patients with a Single Lesion. Ultraschall Med. 2016;37(6):609-18.

43. Wilson SR, Lyshchik A, Piscaglia F, Cosgrove D, Jang HJ, Sirlin C, et al. CEUS LI-RADS: algorithm, implementation, and key differences from CT/MRI. Abdom Radiol (NY). 2018;43(1):127-42.

44. Wang Z, Sheng YY, Dong QZ, Qin LX. Hepatitis B virus and hepatitis $C$ virus play different prognostic roles in intrahepatic cholangiocarcinoma: A meta-analysis. World J Gastroenterol. 2016;22(10): 3038-51.

45. Jeong S, Luo G, Wang ZH, Sha M, Chen L, Xia Q. Impact of viral hepatitis $B$ status on outcomes of intrahepatic cholangiocarcinoma: a meta-analysis. Hepatol Int. 2018;12(4):330-8.

46. Liu W, Chen JR, Hsu CH, Li YH, Chen YM, Lin CY, et al. A zebrafish model of intrahepatic cholangiocarcinoma by dual expression of hepatitis $B$ virus $X$ and hepatitis $C$ virus core protein in liver. Hepatology. 2012;56(6):2268-76.

47. Battaglia S, Benzoubir N, Nobilet S, Charneau P, Samuel D, Zignego AL, et al. Liver cancer-derived hepatitis $C$ virus core proteins shift TGF-beta responses from tumor suppression to epithelialmesenchymal transition. PLoS One. 2009;4(2):e4355.

48. Lee DK, Park SH, Yi Y, Choi SG, Lee C, Parks WT, et al. The hepatitis $\mathrm{B}$ virus encoded oncoprotein $\mathrm{pX}$ amplifies TGF-beta family signaling through direct interaction with Smad4: potential mechanism of hepatitis B virus-induced liver fibrosis. Genes Dev. 2001;15(4):455-66.

49. Xu J, Lamouille S, Derynck R. TGF-beta-induced epithelial to mesenchymal transition. Cell Res. 2009;19(2):156-72.

50. Zou SQ, Qu ZL, Li ZF, Wang X. Hepatitis B virus X gene induces human telomerase reverse transcriptase mRNA expression in cultured normal human cholangiocytes. World J Gastroenterol. 2004;10(15):2259-62.

51. Alison MR, Lovell MJ. Liver cancer: the role of stem cells. Cell Prolif. 2005;38(6):407-21.

52. Roskams T, De Vos R, Van Eyken P, Myazaki H, Van Damme B, Desmet V. Hepatic OV-6 expression in human liver disease and rat experiments: evidence for hepatic progenitor cells in man. J Hepatol. 1998;29(3):455-63.

53. Roskams T, Yang SQ, Koteish A, Durnez A, DeVos R, Huang X, et al. Oxidative stress and oval cell accumulation in mice and humans with alcoholic and nonalcoholic fatty liver disease. Am J Pathol. 2003;
163(4):1301-11.

54. Roskams TA, Libbrecht L, Desmet VJ. Progenitor cells in diseased human liver. Semin Liver Dis. 2003;23(4):385-96.

55. Theise ND, Saxena R, Portmann BC, Thung SN, Yee H, Chiriboga L, et al. The canals of Hering and hepatic stem cells in humans. Hepatology. 1999;30(6):1425-33.

56. Lee JH, Rim HJ, Sell S. Heterogeneity of the "oval-cell" response in the hamster liver during cholangiocarcinogenesis following Clonorchis sinensis infection and dimethylnitrosamine treatment. J Hepatol. 1997;26(6):1313-23.

57. Steinberg P, Steinbrecher R, Radaeva S, Schirmacher P, Dienes HP, Oesch F, et al. Oval cell lines OC/CDE 6 and OC/CDE 22 give rise to cholangio-cellular and undifferentiated carcinomas after transformation. Lab Invest. 1994;71(5):700-9.

58. Andersen JB, Thorgeirsson SS. Genetic profiling of intrahepatic cholangiocarcinoma. Curr Opin Gastroenterol. 2012;28(3):266-72.

59. Komuta M, Spee B, Vander Borght S, De Vos R, Verslype C, Aerts R, et al. Clinicopathological study on cholangiolocellular carcinoma suggesting hepatic progenitor cell origin. Hepatology. 2008;47(5): 1544-56.

60. Gao Q, Zhao YJ, Wang XY, Guo WJ, Gao S, Wei L, et al. Activating mutations in PTPN3 promote cholangiocarcinoma cell proliferation and migration and are associated with tumor recurrence in patients. Gastroenterology. 2014:146(5):1397-407.

61. Zou S, Li J, Zhou H, Frech C, Jiang X, Chu JS, et al. Mutational landscape of intrahepatic cholangiocarcinoma. Nat Commun. 2014; 5:5696.

62. Ahn SM, Jang SJ, Shim JH, Kim D, Hong SM, Sung CO, et al. Genomic portrait of resectable hepatocellular carcinomas: implications of RB1 and FGF19 aberrations for patient stratification. Hepatology. 2014;60(6):1972-82.

63. Schulze K, Imbeaud S, Letouze E, Alexandrov LB, Calderaro J, Rebouissou S, et al. Exome sequencing of hepatocellular carcinomas identifies new mutational signatures and potential therapeutic targets. Nat Genet. 2015;47(5):505-11.

64. Chen Q, Yang LX, Li XD, Yin D, Shi SM, Chen EB, et al. The elevated preoperative neutrophil-to-lymphocyte ratio predicts poor prognosis in intrahepatic cholangiocarcinoma patients undergoing hepatectomy. Tumour Biol. 2015;36(7):5283-9.

65. Lee BS, Lee SH, Son JH, Jang DK, Chung KH, Lee YS, et al. Neutrophil-lymphocyte ratio predicts survival in patients with advanced cholangiocarcinoma on chemotherapy. Cancer Immunol Immunother. 2016;65(2):141-50.

66. Okuno M, Ebata T, Yokoyama Y, Igami T, Sugawara G, Mizuno T, et al. Evaluation of inflammation-based prognostic scores in patients undergoing hepatobiliary resection for perihilar cholangiocarcinoma. J Gastroenterol. 2016;51(2):153-61.

67. Lim CJ, Lee YH, Pan L, Lai L, Chua C, Wasser M, et al. Multidimensional analyses reveal distinct immune microenvironment in hepatitis B virus-related hepatocellular carcinoma. Gut. $2018 \mathrm{Jul} 3$. pii: gutjnl-2018-316510. doi: 10.1136/gutjnl-2018-316510. [Epub ahead of print]

68. Wei Y, Gao F, Zheng D, Huang Z, Wang M, Hu F, et al. Intrahepatic cholangiocarcinoma in the setting of HBV-related cirrhosis: Differentiation with hepatocellular carcinoma by using Intravoxel incoherent motion diffusion-weighted MR imaging. Oncotarget. 2018;9(8):7975-83. 